\title{
Eclipsing binary asteroid 90 Antiope
}

\author{
T. Michałowski ${ }^{1}$, P. Bartczak ${ }^{1}$, F. P. Velichko ${ }^{2,3}$, A. Kryszczyńska ${ }^{1}$, T. Kwiatkowski ${ }^{1}$, S. Breiter ${ }^{1}$, \\ F. Colas ${ }^{4}$, S. Fauvaud ${ }^{5}$, A. Marciniak ${ }^{1}$, J. Michałowski ${ }^{6}$, R. Hirsch ${ }^{1}$, R. Behrend ${ }^{7}$, \\ L. Bernasconi ${ }^{8}$, C. Rinner ${ }^{9}$, and S. Charbonnel ${ }^{10}$ \\ 1 Astronomical Observatory, Adam Mickiewicz University, Słoneczna 36, 60-286 Poznań, Poland \\ e-mail: tmich@amu.edu.pl \\ 2 Research Institute of Astronomy, Kharkiv Karazin National University, Sums'ka 35, 61022 Kharkiv, Ukraine \\ 3 Isaac Newton Institute of Chile, Crimean Branch, Chile \\ ${ }^{4}$ Institut de Mécanique Céleste, 77 Av. Denfert Rochereau, 75014 Paris, France \\ 5 Astroqueyras Association, Mairie, 05350 Saint Veran, France \\ ${ }^{6}$ Poznań University of Technology, Poznań, Poland \\ 7 Geneva Observatory, 1290 Sauverny, Switzerland \\ 8 Les Engarouines Observatory, France \\ 9 Ottmarsheim Observatory, France \\ ${ }^{10}$ Durtal Observatory, France
}

Received 16 March 2004 / Accepted 11 May 2004

\begin{abstract}
CCD observations of the binary asteroid 90 Antiope were carried out at seven observatories (Borowiec, Kharkiv, Pic du Midi, Chateau Renard, Les Engarouines, Ottmarsheim, and Durtal) on 31 nights from December 2002 through April 2003. The results show two-component lightcurves with each showing the same period of $16.505 \pm 0.002 \mathrm{~h}$. The first component is associated with the rotation of the two non-spherical bodies of the system; the second one is due to eclipses/occultations in the binary system. The lightcurves suggest that Antiope is an 'almost synchronous system' with orbital period of $16.5051 \pm 0.0002 \mathrm{~h}$ and a little shorter rotational one of $16.5047 \pm 0.0002 \mathrm{~h}$. The ecliptic coordinates of the pole of the orbit system are $\lambda_{n}=17^{\circ} \pm 5^{\circ}$ and $\beta_{n}=25^{\circ} \pm 5^{\circ}$. A possibility of the observation of the eclipsing events during two future oppositions (April 2004 and July 2005) has been predicted.
\end{abstract}

Key words. minor planets, asteroids

\section{Introduction}

In the last decade, it has been shown that satellites of asteroids do exist. Observations and modelling of rotational characteristics of binary systems are very important for our understanding of asteroid composition and collisional evolution. They allow a determination of the bulk density of these bodies - a parameter that is otherwise very difficult to obtain.

The question in the title of the paper "Do Asteroids Have Satellites?" (Weidenschilling et al. 1989) published in Asteroids II has been recently answered in Asteroids III with the paper "Asteroids Do Have Satellites" (Merline et al. 2002a). In the latter paper we find a complete list of discoveries up to August 2002 (see references therein for details). The authors reported 16 binary asteroids among main belt, outer main belt, Trojan, and Transneptunian objects discovered by spacecraft (243 Ida), adaptive optics, HST and ground-based direct imaging.

Satellites have been also detected for near-Earth asteroids. Merline et al. (2002a) reported 6 such objects discovered by radar and 11 binaries confirmed by their lightcurves.
Photometric observations can indicate binary asteroids when they show two-period lightcurves. Usually the shorter periods are associated with the rotations of primary objects while the longer ones with orbital rotations and/or rotations of secondaries when they are synchronous. Moreover, some twoperiod-lightcurve NEAs (1994 AW1, 1991 VH, and 1996 FG3 - see appropriate references given in Merline et al. (2002a) for details) show that their long-period components clearly have occultation-like features.

The binary asteroids from the NEA population share some similar features. They are all small objects with primary diameters of $0.7-4.0 \mathrm{~km}$ and with secondary-to-primary diameter ratios in the range of 0.2-0.6. They all are inner planet-crossers approching the orbits of Earth and Venus. The primary components of these systems are fast rotators with periods of 2.3-3.6 $\mathrm{h}$ and low amplitudes of $0.1-0.2 \mathrm{mag}$, suggesting nearly spheroidal shapes. Orbit semimajor axes are in the range 3.4-6.6 primary radii and eccentricities seem to be low, less than 0.1 . It is said that about $16 \%$ of NEAs are binary.

The main belt and outer main belt binary asteroids are different to NEA binaries. With the exception of 90 Antiope (see 
next section) the rotation periods of primaries are 4-6 h and amplitudes are larger by up to $0.25 \mathrm{mag}$. The satellites are only a few kilometers in diameter, which makes them much smaller than the primary bodies of the systems. For most of them the orbit semimajor axes are about 10 primary radii.

Transneptunian binary objects seem to be different. The primaries are about $100 \mathrm{~km}$ in radius with similarly sized secondaries. However, the orbits are of 10000-100 $000 \mathrm{~km}$, which means that the semimajor axes are about $100-1000$ primary radii.

The differences between these three populations of binary asteroids might be a result of the different origins of such systems. Merline et al. (2002a) gave a review of the mechanisms of origin and evolution of binary systems (see also references therein). One of them, efficient for NEAs, can be a close planetary encounter that subjects an asteroid to tidal stress and torques that may produce a binary. For main-belt binaries, which are fast rotators having rather large amplitudes, and the mass ratios of $10^{-3}$, the impact ejecta seem to be the mechanism of formation of small satellites. Many binaries are members of dynamical families, being fragments of larger parent bodies disrupted by catastrophic collisions. Some fragments may move around each other, forming a binary system. Also, binary nature of some asteroids can be primordial. This can be true for the binaries with size ratios close to unity and having large separations. This means that we should expect such systems among Trojans and Transneptunian objects.

The list of binary asteroids presented by Merline et al. (2002a) contains the detections made before August 2002. New discoveries of satellites have been made since then. Using adaptive optics systems, it was announced that 121 Hermione, 1509 Escalangona, 283 Emma, 379 Huenna, and 130 Elektra have satellites (Merline et al. 2002b, 2003a,b; Margot 2003; Merline et al. 2003c, respectively). Also new Transneptunian binaries 2001 QC298 (Noll et al. 2002) and 2000 CQ114 (Stephens et al. 2004) discovered by HST were reported. Ground-based direct images confirmed the binary nature of TNO 2003 UN284 (Millis 2003). Radar observations revelead that the near-Earth asteroid 5381 Sekhmet, 2003 SS84, 69230 Hermes, and 1990 OS are binary (Nolan et al. 2003a; Nolan et al. 2003b ; Margot et al. 2003; Ostro et al. 2003, respectively). Two binary NEAs 660631998 RO1 and 65803 1996 GT (Pravec et al. 2003a,b) were confirmed by radar and mutual eclipses/occultations visible in their lightcurves. Two members of the Koronis family 228991999 TO14 and 172462000 GL74 were discovered to be binary by using HST images (Merline et al. 2003d; Tamblyn et al. 2004).

Veillet et al. (2002) analyzed dynamics of the binary Transneptunian object 1998 WW31, the first discovered double object (beside the Pluto/Charon pair) among this group of bodies. They predicted a possibility of mutual eclipses in this system of long orbital period (570 days). If these phenomena are seen from the Earth, this object will be the first eclipsing TNO.

Eclipsing binaries have also been discovered among Main Belt Asteroids. Ryan (2003) reported photometric observations of the asteroid 3782 Celle. He observed this object in September 2001, and from December 2002 to February 2003. The asteroid showed a normal rotational lightcurve (period of $3.84 \mathrm{~h}$ and amplitude $0.10-0.15 \mathrm{mag}$ ) superimposed on deeper attenuation events that varied in amplitude from 0.15 to $0.30 \mathrm{mag}$. The attenuations were of two distinct types that could be identified as primary and secondary occultations/eclipses. This showed that 3782 Celle is an asynchronous binary system with a secondary-to-primary diameter ratio of 0.42 and an orbital period of $36.57 \mathrm{~h}$. Since this asteroid has been associated with the Vesta family, this is the first identified binary among the so-called "Vesta-chips". The asteroid 3782 Celle is really the first main-belt binary system discovered by its eclipsing lightcurves as Hansen et al. (1997) did not make such an interpretation of their observations of 90 Antiope (see next section).

The asteroid 1089 Tama was observed photometrically in December 2003 and January 2004 (Behrend et al. 2004a). The lightcurve of the amplitude $0.38 \mathrm{mag}$ was superimposed with eclipses/occultations with brightness drop of $0.5 \mathrm{mag}$ in the same period of $16.445 \mathrm{~h}$. This asteroid seems to be a synchronous system with a secondary-to-primary diameter ratio of about 0.7 .

The observations carried out in February 2004 showed that the asteroid 1313 Berna was also a synchronized binary with similar-sized components (Behrend et al. 2004b). The rotational and orbital periods are equal to $25.464 \mathrm{~h}$ and the amplitude caused by the rotation is 0.25 mag while that from the eclipses/occultations is of $0.7 \mathrm{mag}$.

For most binary asteroids, it is possible to determine their bulk density. This parameter can be as small as $0.8 \mathrm{~g} / \mathrm{cm}^{3}$ for NEA 2000 UG11 or $1.2 \mathrm{~g} / \mathrm{cm}^{3}$ for 45 Eugenia, and can reach $2.6 \mathrm{~g} / \mathrm{cm}^{3}$ for NEA $1999 \mathrm{KW} 4$ and 243 Ida (see Merline et al. 2002a for a list of available results).

\section{Previous works on the asteroid 90 Antiope}

The first lightcurves of 90 Antiope were obtained by Hansen et al. (1997) on four nights in December 1996. The rotational period was determined as $16.509 \mathrm{~h}$ and the amplitude was about $0.70 \mathrm{mag}$. The light variation was similar to a typical lightcurve of an eclipsing binary star. However, they did not make this interpretation.

The binary nature of this asteroid was discovered in August 2000 by Merline et al. (2000). Using the Keck Adaptive Optics system, they found that 90 Antiope was a double asteroid with similar-sized components of diameter $85 \mathrm{~km}$ separated by $170 \mathrm{~km}$, rather than a single body of size $120 \mathrm{~km}$ as reported in IRAS data (http://pdssbn.astro.umd.edu/). The orbital period of the components was found to be $16.5 \mathrm{~h}$, consistent with the period derived from the 1996 photometric observations. The distance between these two bodies and the orbital period indicate a mass of $4.12 \times 10^{20} \mathrm{~g}$ for each of them. Thus, the density is $1.3 \mathrm{~g} / \mathrm{cm}^{3}$.

As Antiope is a member of the Themis family, Weidenschilling et al. (2001) expected that the binarity of this asteroid could not be primordial. It was probably formed during or after breakup of the parent body forming the Themis family. However, they concluded that the formation of such a large binary system was a highly improbable event. According to their conclusion Antiope should be unique. 
Table 1. Observatories, telescopes, CCD cameras and observers.

\begin{tabular}{|c|c|c|c|c|}
\hline Code & Observatory & Telescope & CCD camera & Observers / Reducers \\
\hline 1 & Borowiec, Poland & $0.40 \mathrm{~m}$ & SBIG ST-7 & $\begin{array}{l}\text { T. Michałowski, Kwiatkowski, Kryszczyńska, } \\
\text { Marciniak, J. Michałowski, Hirsch }\end{array}$ \\
\hline 2 & Kharkiv, Ukraine & $0.70 \mathrm{~m}$ & SBIG ST-6V & Velichko \\
\hline 3 & Pic du Midi, France & $1.05 \mathrm{~m}$ & Thomson 7863 & Colas, Kryszczyńska \\
\hline 4 & Chateau Renard, France & $0.62 \mathrm{~m}$ & KAF 400 & Fauvaud \\
\hline 5 & Les Engarouines, France & $0.21 \mathrm{~m}$ & KAF 1600 & Bernasconi, Behrend \\
\hline 6 & Ottmarsheim, France & $0.30 \mathrm{~m}$ & SBIG ST-8e & Rinner, Behrend \\
\hline 7 & Durtal, France & $0.30 \mathrm{~m}$ & KAF 400E & Charbonnel, Behrend \\
\hline
\end{tabular}

Most of the co-authors of the present paper observed 90 Antiope in the 2000 (Michałowski et al. 2001, hereafter Paper I) and the 2001/02 (Michałowski et al. 2002, Paper II) apparitions.

We obtained lightcurves of this asteroid on 14 nights in September-November 2000 (Paper I). A synodical period of $16.496 \mathrm{~h}$ (consistent with that found by Hansen et al. 1997) and an observed amplitude of brightness variation of 0.08 mag were found. This small amplitude was due to the rotation of noncircular components of the binary system rather than to mutual eclipses/occultations. The lightcurve was asymmetrical as the interval between two maxima (and minima, respectively) was larger than a half of the rotational cycle.

From October 2001 until the beginning of February 2002, Antiope was observed on 26 nights (Paper II). The asteroid displayed a two-component lightcurve with each component showing the same period of $16.505 \mathrm{~h}$, consistent with previously-published values, as presented above. The first component was associated with the rotation of two non-spherical bodies (at least one of them was non-spherical) giving a socalled rotational lightcurve; the second one, with two sharp minima, was due to mutual occultations/eclipses in the binary system (eclipsing lightcurve). One of the eclipsing minima ( $m 2$ in the figures) occurred in the same phase as the shallow rotational minimum. The eclipsing minima were always a half period apart (which indicates circular orbits of both components), so $m l$ did not appear exactly at the phase of the deeper rotational minimum. The total lightcurve of Antiope was a sum of the symmetrical eclipsing lightcurve and the asymmetrical rotational one. We also noticed that the rotational amplitude of 0.10 mag was almost constant during the observational interval. The values of the symmetrical eclipsing amplitude $(\mathrm{m} 2)$ were in the range of $0.05-0.12 \mathrm{mag}$, depending linearly on phase angle as it changed from $0.6 \mathrm{deg}$ to $15 \mathrm{deg}$. The slope of this relationship seemed to be $0.005 \mathrm{mag} / \mathrm{deg}$.

In the time of preparing Paper II we had lightcurves of Antiope from two oppositions $(1996,2001 / 02)$ with occultations/eclipses (Hansen et al. 1997; and Paper II, respectively) and one (2000) with no eclipses (Paper I). The whole amplitude in 1996 was 0.70 mag and it was suggested that it had been caused by a total occultation of two equal bodies. Assuming this and using the 1996 and 2001/02 data we could determine the orientation of the system's orbit from the analysis of both amplitudes and duration of the eclipses/occultations. The results from these two methods (amplitude and timing) differed a little but showed that we should expect a larger eclipsing amplitude in the 2003 apparition than during the 2001/02 one. Also, an observer should not see eclipses/occultations in April 2004 and quite large eclipsing amplitude in July 2005. These predictions depend on the assumption that there were total eclipses/occultations in December 1996 and no precession in the system.

\section{CCD photometry in the $2002 / 03$ apparition}

We started our observations of Antiope in December 2002 and performed them on 31 nights until the beginning of April 2003. The summary of the instruments and observers/reducers is shown in Table 1. Most of these data were obtained in the same observatories (Nos 1-4 in Table 1) as those described in Paper II. Some additional lightcurves were obtained at three small observatories in France (Nos 5-7). Details on these observatories and reduction procedures can be found at the web site http: //obswww . unige.ch/ behrend/

The aspect data of the asteroid are listed in part of Table 2. The columns give the date of the observation referring to the mid-time of the observed lightcurve, asteroid-Sun $(r)$ and asteroid-Earth $(\Delta)$ distances (in A.U.), solar phase angle, ecliptic longitude $(\lambda)$ and latitude $(\beta)$ for the $\mathrm{J} 2000$ epoch, and the code of the observatory as given in Table 1 .

The observations are presented as composite lightcurves in Figs. 1-5. The vertical shift of each lightcurve was obtained by minimizing the dispersion of data points relative to their neighbours. The abscissa is the rotational phase with zero point corrected for light-time. The observed minima $(m 1, m 2)$ are indicated in the graphs.

As expected from the prediction given in Paper II, the asteroid 90 Antiope showed a two-component lightcurve, confirming the period of $16.505 \pm 0.002 \mathrm{~h}$. However, we noticed some unexpected differences. The eclipsing amplitude was much smaller than 0.34-0.43 mag predicted in Paper II. The occultations $m 2$ were clearly visible only in December 2002 (Fig. 1) and March-April 2003 (Fig. 5) when the asteroid was far from its opposition and phase angle was in the range of $13^{\circ}-15^{\circ}$ for these observations. The value of the eclipsing amplitude during these two runs was about 0.05 mag. When the phase angle was smaller, in January 2003 (Fig. 2) and at the end of February 2003 (Fig. 4), the $m 2$ eclipsing amplitude decreased. It was so small that we had problems recognizing it because of the noisy lightcurves. At the beginning of February 2003 (Fig. 3), when the phase angle reached its minimum below $1^{\circ}$, the $m 2$ eclipsing amplitude dropped practically to zero. A similar 
Table 2. Aspect data and times of observed minima of 90 Antiope.

\begin{tabular}{|c|c|c|c|c|c|c|c|c|}
\hline \multirow[t]{2}{*}{ Date (UT) } & $r$ & $\Delta$ & $\begin{array}{c}\text { Phase } \\
\text { angle }\end{array}$ & \multicolumn{2}{|c|}{$(\mathrm{J} 2000)$} & \multirow[t]{2}{*}{ Obs. } & \multicolumn{2}{|c|}{$\begin{array}{c}m 1 \quad m 2 \\
\text { Julian Days }\end{array}$} \\
\hline & $(\mathrm{AU})$ & $(\mathrm{AU})$ & $\left({ }^{\circ}\right)$ & $\left({ }^{\circ}\right)$ & $\left({ }^{\circ}\right)$ & & +245 & 2000 \\
\hline 2002 Dec. 10.1 & 3.655 & 3.142 & 14.3 & 143.8 & 2.2 & 1 & 618.5606 & \\
\hline 2002 Dec. 11.0 & 3.655 & 3.129 & 14.2 & 143.8 & 2.2 & 1 & & \\
\hline 2002 Dec. 13.1 & 3.655 & 3.101 & 13.9 & 143.9 & 2.3 & 1,3 & & 621.6682 \\
\hline 2002 Dec. 15.1 & 3.655 & 3.074 & 13.6 & 143.8 & 2.3 & 2,3 & 623.3748 & 623.7318 \\
\hline 2002 Dec. 16.1 & 3.655 & 3.061 & 13.5 & 143.8 & 2.3 & 2 & & 624.4196 \\
\hline 2003 Jan. 01.1 & 3.655 & 2.872 & 10.5 & 143.0 & 2.5 & 1 & 640.5626 & \\
\hline 2003 Jan. 06.0 & 3.655 & 2.823 & 9.4 & 142.4 & 2.6 & 4 & & \\
\hline 2003 Jan. 07.2 & 3.655 & 2.812 & 9.1 & 142.3 & 2.6 & 4 & 646.7443 & \\
\hline 2003 Jan. 09.0 & 3.655 & 2.797 & 8.6 & 142.1 & 2.6 & 1 & & 648.5018 \\
\hline 2003 Jan. 10.0 & 3.654 & 2.788 & 8.4 & 141.9 & 2.6 & 2,4 & & \\
\hline 2003 Jan. 11.1 & 3.654 & 2.779 & 8.1 & 141.8 & 2.6 & 1,4 & & 650.5626 \\
\hline 2003 Jan. 12.2 & 3.654 & 2.770 & 7.8 & 141.6 & 2.6 & 4 & 651.5742 & \\
\hline 2003 Feb. 01.1 & 3.652 & 2.672 & 1.9 & 138.2 & 2.8 & 1 & 671.5178 & \\
\hline 2003 Feb. 02.0 & 3.652 & 2.671 & 1.6 & 138.0 & 2.8 & 1 & & 672.5860 \\
\hline 2003 Feb. 08.0 & 3.651 & 2.666 & 0.9 & 136.8 & 2.8 & 3 & 678.3953 & \\
\hline 2003 Feb. 09.0 & 3.651 & 2.667 & 1.1 & 136.6 & 2.8 & 3 & & 679.4633 \\
\hline 2003 Feb. 22.9 & 3.649 & 2.703 & 5.3 & 134.0 & 2.8 & 1 & 693.5211 & \\
\hline 2003 Feb. 23.9 & 3.648 & 2.708 & 5.6 & 133.8 & 2.8 & 1 & & 694.5558 \\
\hline 2003 Feb. 24.9 & 3.648 & 2.713 & 5.9 & 133.7 & 2.8 & 1 & 695.5838 & \\
\hline 2003 Feb. 25.9 & 3.648 & 2.719 & 6.2 & 133.5 & 2.8 & 1 & 696.2713 & 696.6259 \\
\hline 2003 Mar. 08.9 & 3.645 & 2.796 & 9.2 & 131.9 & 2.7 & 5 & & \\
\hline 2003 Mar. 16.9 & 3.643 & 2.870 & 11.1 & 131.1 & 2.7 & 2 & & \\
\hline 2003 Mar. 19.8 & 3.642 & 2.900 & 11.7 & 130.8 & 2.7 & 2 & 718.2809 & \\
\hline 2003 Mar. 22.9 & 3.641 & 2.934 & 12.3 & 130.6 & 2.6 & 5 & & 721.3828 \\
\hline 2003 Mar. 23.9 & 3.641 & 2.946 & 12.5 & 130.6 & 2.6 & 6 & 722.4061 & \\
\hline 2003 Mar. 25.8 & 3.641 & 2.967 & 12.8 & 130.5 & 2.6 & 2 & & \\
\hline 2003 Mar. 28.8 & 3.640 & 3.003 & 13.4 & 130.4 & 2.6 & 2 & 727.2200 & \\
\hline 2003 Apr. 02.8 & 3.638 & 3.065 & 14.1 & 130.3 & 2.5 & 2 & & 732.3849 \\
\hline 2003 Apr. 03.9 & 3.638 & 3.079 & 14.2 & 130.3 & 2.5 & 5 & 733.4105 & \\
\hline 2003 Apr. 04.9 & 3.637 & 3.092 & 14.4 & 130.3 & 2.5 & 5 & & 734.4499 \\
\hline 2003 Apr. 07.9 & 3.636 & 3.132 & 14.7 & 130.3 & 2.5 & 2,7 & 737.5354 & 737.2033 \\
\hline
\end{tabular}

Observatory code given in Table 1.

behaviour could be observed for the $m l$ eclipsing amplitude. Its value decreases for smaller phase angles. In this case the occultations were clearly visible even for the smallest phase angle as in Fig. 3. The rotational amplitude (i.e. without occultation) changed a little from 0.09 mag for the largest phase angle (Figs. 1 and 5) to 0.07 mag for the smallest one (Fig. 3).

The duration of $m 2$ eclipsing minima is about $2 \mathrm{~h}$. It can be determined from the December and March-April data (Figs. 1 and 5) as they are clearly visible.

Moreover, we have noticed one more difference not expected before. As mentioned in Paper II, $m 2$ of the eclipsing lightcurve occurred in the same phase as the shallow rotational minimum. This allowed us to conclude that rotational periods of both bodies were equal to the orbital one, which would indicate the synchronous rotation.

Antiope did not show a similar property in the 2002/03 apparition. All presented figures are constructed in such way that $m 1$ and $m 2$ eclipsing minima occur at 0.25 and 0.75 rotational phase. However, in Fig. 3 when the $m 2$ eclipsing minimum disappeared, we could see a minimum at the 0.8 rotational phase. So, we observed some shift between two components of the lightcurve. This shift is probably not due to a change in the direction of sunlight, as it is the same in Fig. 1 and Fig. 5, when the asteroid was before and after its opposition, respectively. The observed $m l$ events are the sum of symmetrical eclipsing minima and asymmetrical rotational ones. The shift between these two components made the $m l$ events look more symmetrical and wider than those observed in 2001/02.

The times (corrected for light-time) of the observed $m 1$ and $m 2$ minima are displayed in Table 2. For the lightcurves from the beginning of February the times for $m 2$ are not for eclipsing minima.

A summary of the photometric results for Antiope is presented in Table 3 . The dates and ecliptic coordinates of the asteroid for the two next oppositions are also given.

\section{Modelling the eclipsing amplitudes}

The eclipsing amplitudes depend on an inclination $i$ of the orbit of the double asteroid system. We can easily calculate the values of these amplitudes for Antiope assuming the diameters of the components ( $85 \mathrm{~km}$ for each) and the separation 


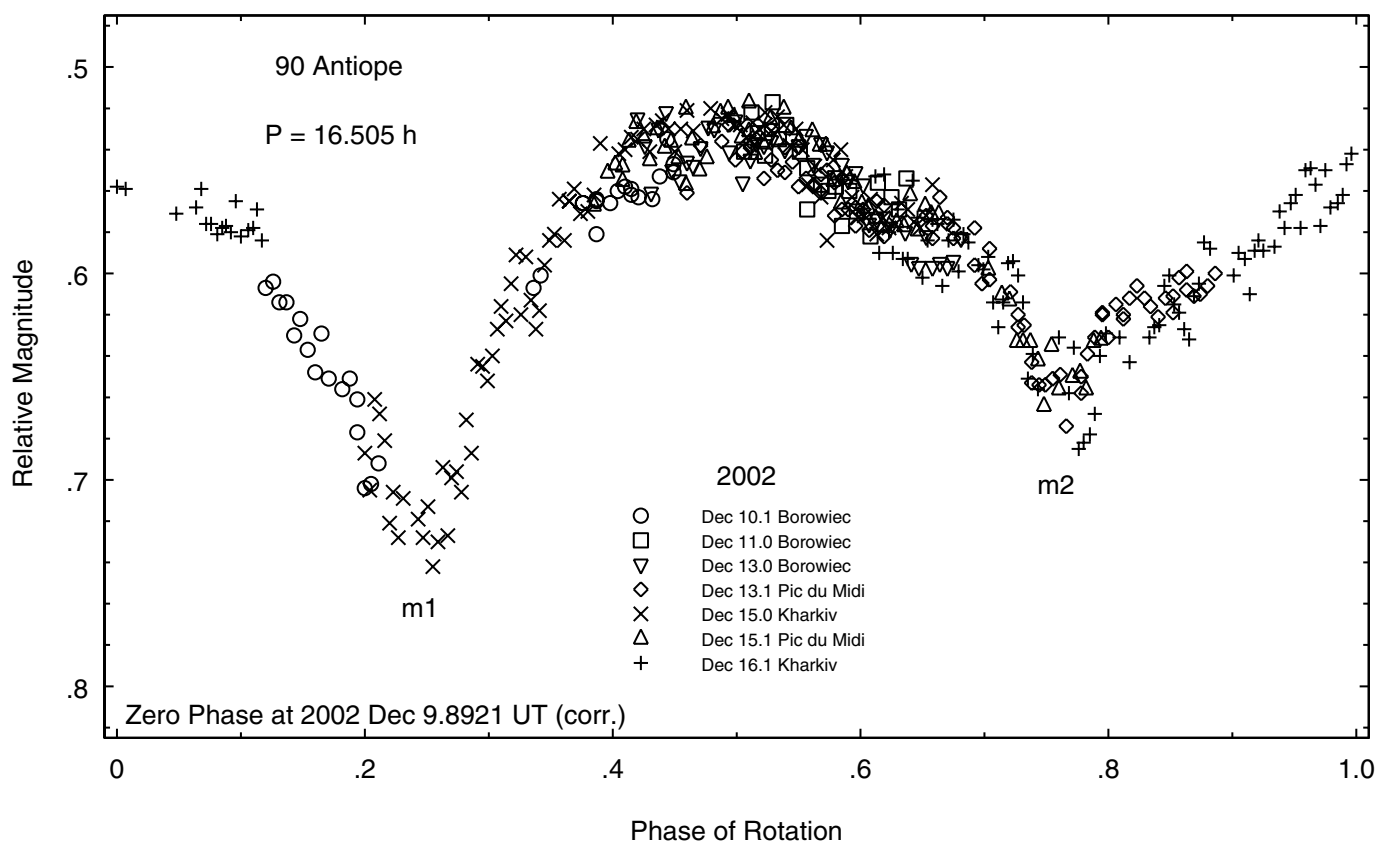

Fig. 1. Composite lightcurve of 90 Antiope in December 2002.

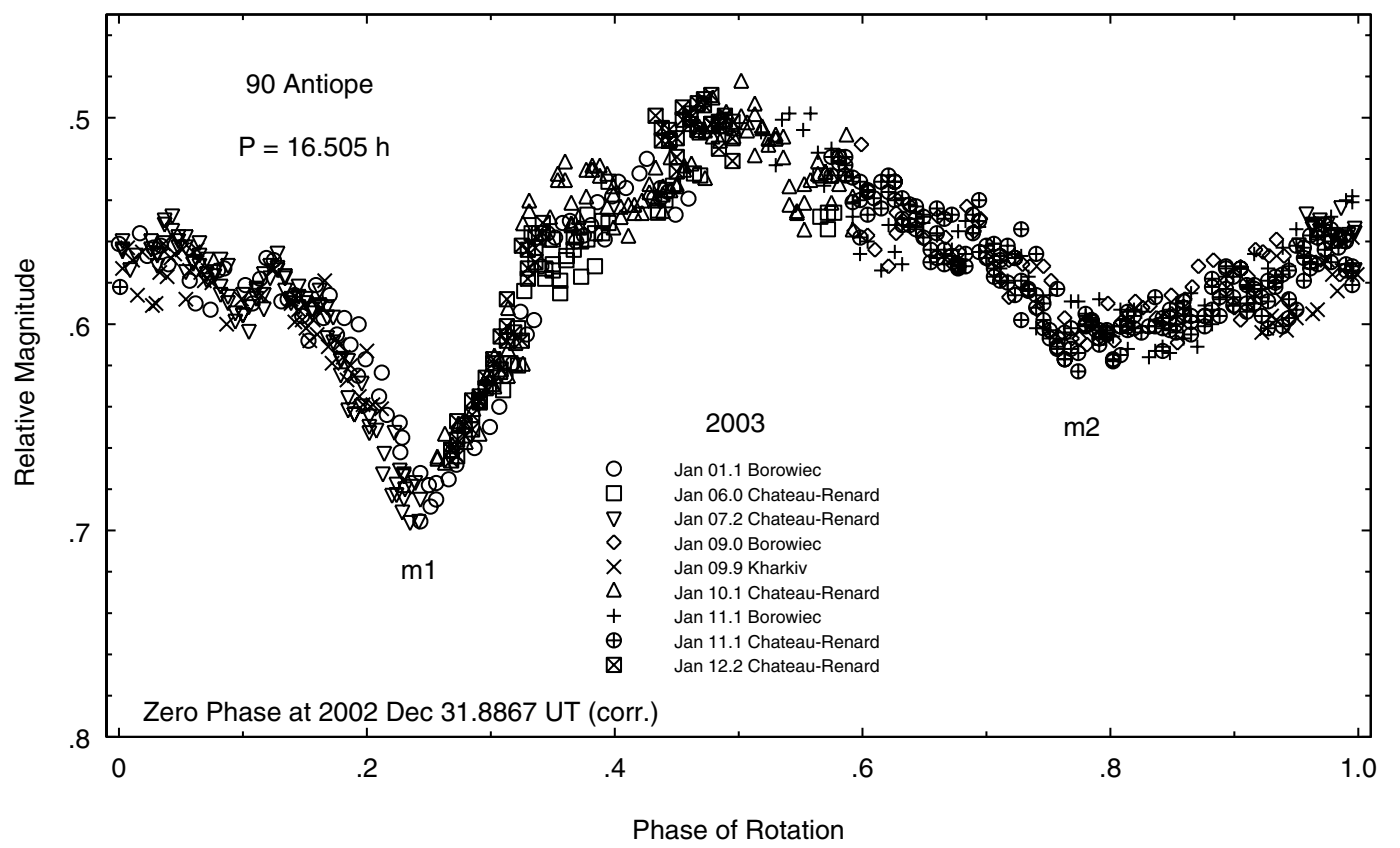

Fig. 2. Composite lightcurve of 90 Antiope in January 2003.

between them to be $170 \mathrm{~km}$ (see also Papers I and II). The central eclipse/ocultation can be expected when $i=90^{\circ}$, which occurs when $\lambda-\lambda_{n}=90^{\circ}$ or $\beta_{n}=90^{\circ}$, where $\left(\lambda_{n}, \beta_{n}\right)$ are the ecliptic coordinates of the pole of the system orbit, and $\lambda$ is geocentric ecliptic longitudes of the asteroid. For Antiope, because of similar-sized components, the amplitude of the central eclipse/occultation should be $0.75 \mathrm{mag}$.

The lines in Fig. 6 represent the calculated amplitudes versus $\lambda-\lambda_{n}$. Because of the symmetry, the calculations have been performed for $\lambda-\lambda_{n}$ within the range $0^{\circ}-180^{\circ}$. The modelled amplitudes have been calculated for various $\beta_{n}$ shown with the step of $10^{\circ}$. The curves for $0^{\circ}, 10^{\circ}$, and $20^{\circ}$ are so close to the one for $30^{\circ}$ that they are not shown. It can be concluded that the inclination of the system orbit should be less than $60^{\circ}$ as we observed Antiope without eclipsing events in 2000 (Paper I).

It was expected to obtain $\left(\lambda_{n}, \beta_{n}\right)$ by comparing the observed eclipsing amplitude with modelled ones. We have plotted the observed amplitudes for the smallest phase angles (see Table 3) against the ecliptic longitudes of the asteroid. Because of the symmetry, the 2000 opposition was taken with longitude of $175^{\circ}$ (instead of $355^{\circ}$ as shown in Table 3 ). The horizontal shift between these two plots should give us the position at which all the observational amplitudes coincide with one curve calculated for a particular value of $\beta_{n}$. Unfortunately, we 


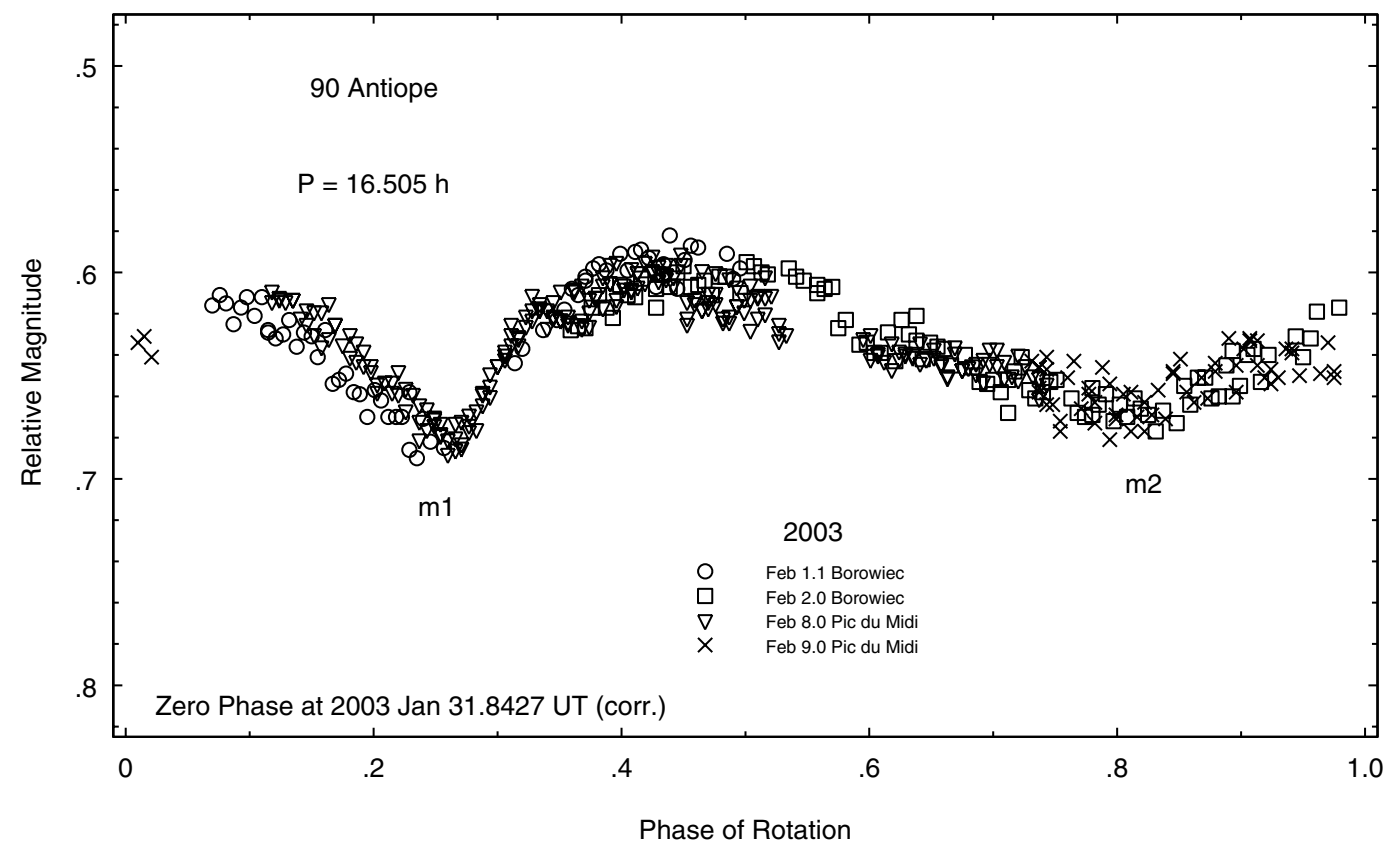

Fig. 3. Composite lightcurve of 90 Antiope at the beginning of February 2003.

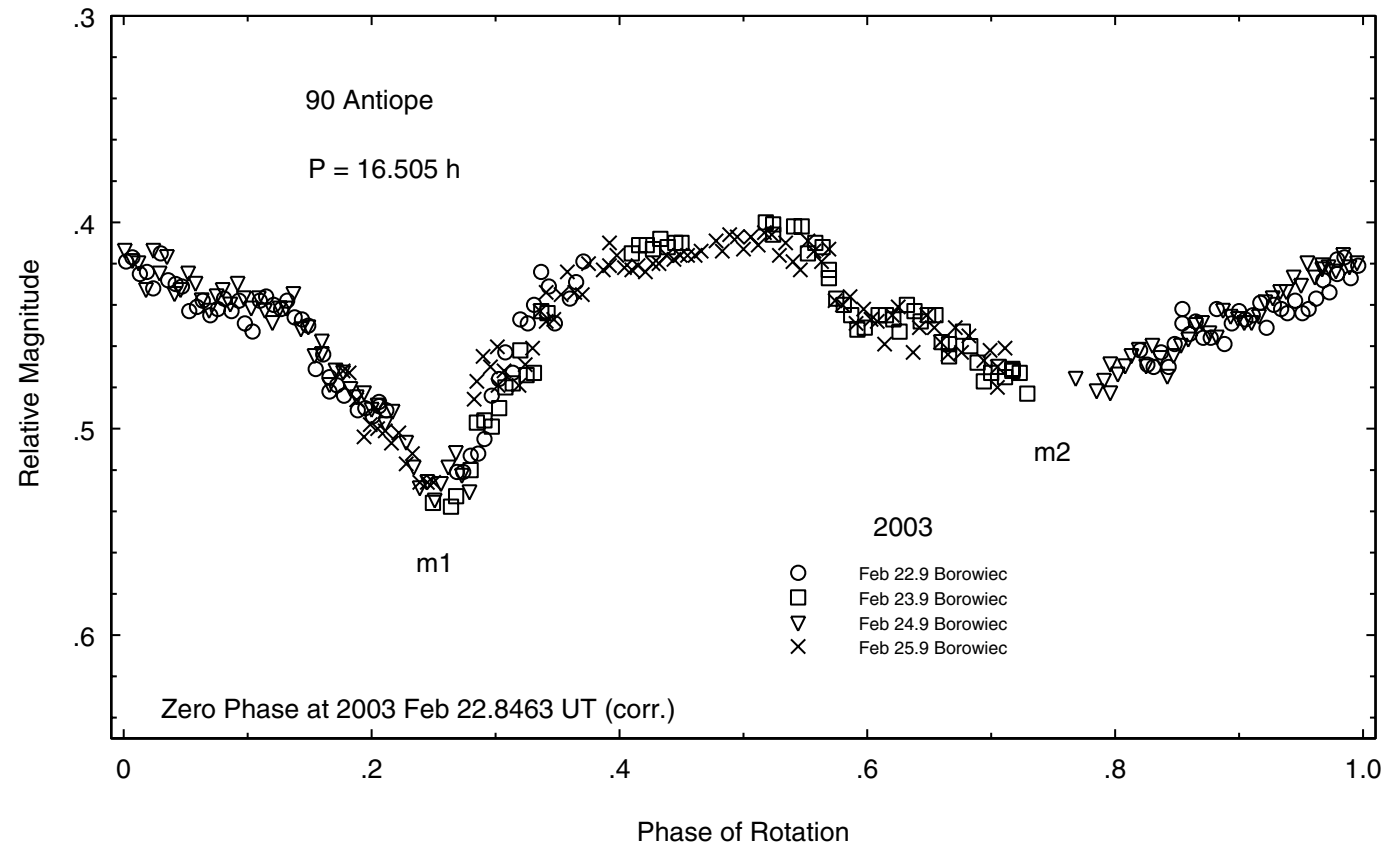

Fig. 4. Composite lightcurve of 90 Antiope at the end of February 2003.

could not find such a position. It has been only possible to find the shift, which indicates $\lambda_{n}=23^{\circ}$, that the points for 1996 and 2001 show $\beta_{n}=30^{\circ}-40^{\circ}$, with the 2000 point outside the eclipsing region. If the 2003 observations would confirm a similar value of $\beta_{n}$, than the eclipsing amplitude for the zero solar phase angle was about $0.15 \mathrm{mag}$, much bigger than we observed.

The existing discrepancy between the observed and modelled eclipsing amplitudes (Fig. 6) indicates that the model assumed for Antiope is not good. Contrary to our assumption, the system of Antiope might not be synchronous and/or the precession in the system should be taken into account.

\section{Modelling the lightcurves of Antiope}

Looking for the explanation of the observed brightness variations and for the means of predicting the lightcurve amplitudes in future oppositions, we have attempted to simulate the lightcurve of Antiope using a simple model. We have assumed that the system consists of a spheroid and a sphere. This is promising, because the dynamics of the system, while 


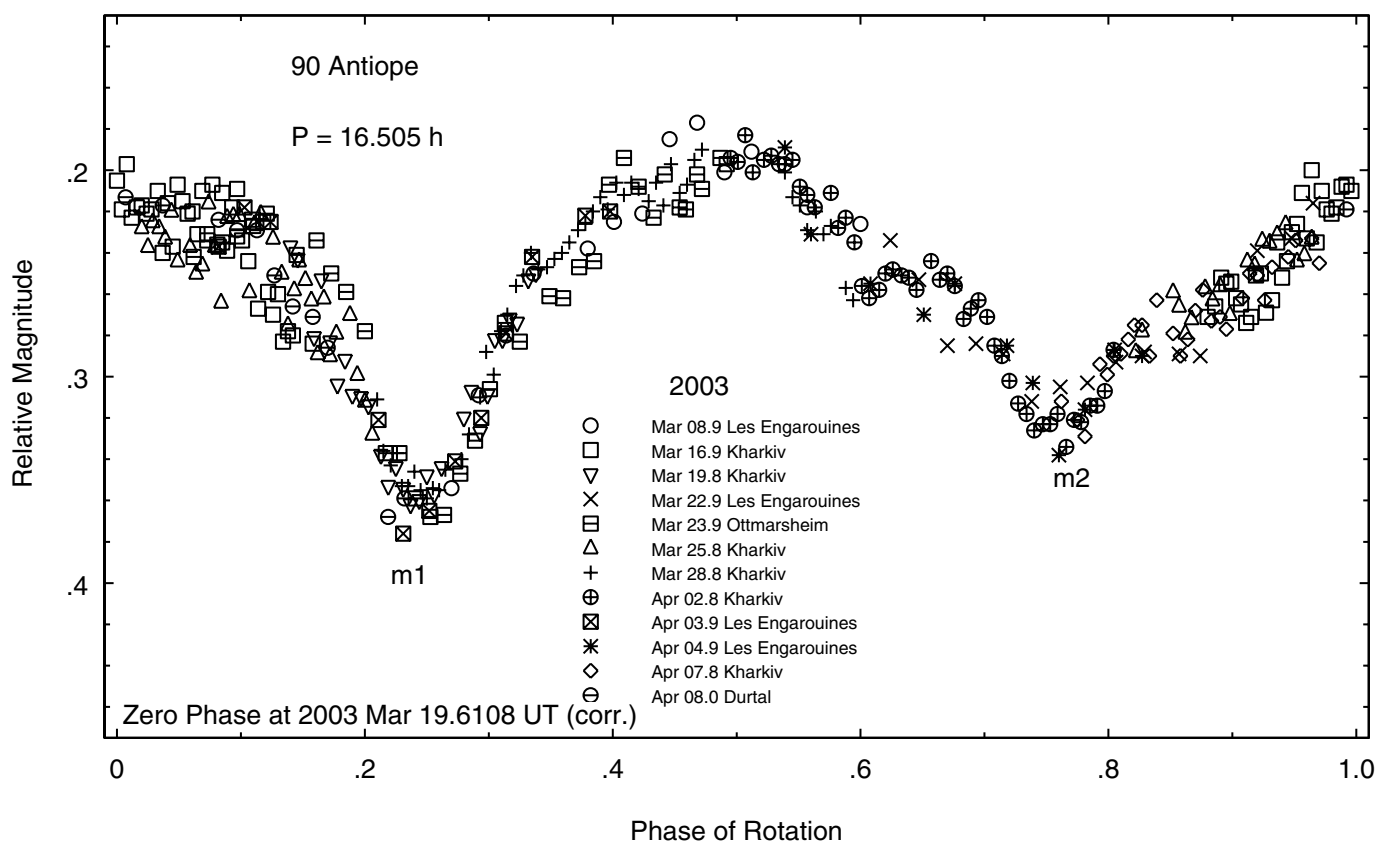

Fig. 5. Composite lightcurve of 90 Antiope in March-April 2003.

Table 3. Summary of photometric results for 90 Antiope.

\begin{tabular}{|c|c|c|c|c|c|c|c|c|}
\hline $\begin{array}{l}\text { Interval of } \\
\text { observation }\end{array}$ & $\begin{array}{l}\text { Number } \\
\text { of nights }\end{array}$ & $\begin{array}{r}\lambda \\
{\left[{ }^{\circ}\right]}\end{array}$ & $\begin{array}{c}\beta \\
{\left[{ }^{\circ}\right]}\end{array}$ & $\begin{array}{c}\text { Eclipse/ } \\
\text { occultation }\end{array}$ & $\begin{array}{c}\text { Rotational } \\
\text { amplitude } \\
\text { [mag] }\end{array}$ & $\begin{array}{c}\text { Eclipsing } \\
\text { amplitude } \\
\text { [mag] }\end{array}$ & $\begin{array}{l}\text { Duration } \\
\text { of eclipse }\end{array}$ & Reference \\
\hline 14-22 Dec. 1996 & 4 & 120 & 2 & Yes & 0.14 & 0.56 & $2^{\mathrm{h}} 50^{\mathrm{m}}$ & Hansen et al. (1997) \\
\hline 29 Sep.-20 Nov. 2000 & 14 & 355 & -3 & No & 0.08 & - & - & Paper I \\
\hline 20 Oct. $2001-8$ Feb. 2002 & 26 & 80 & 1 & Yes & 0.10 & $0.05-0.12$ & $2^{\mathrm{h}} 10^{\mathrm{m}}$ & Paper II \\
\hline 10 Dec. $2002-8$ Apr. 2003 & 31 & 137 & 3 & Yes & $0.07-0.09$ & $0.00-0.05$ & $2^{\mathrm{h}} 00^{\mathrm{m}}$ & Present work \\
\hline Apr. 2004 & & 197 & 3 & No? & & & & \\
\hline Jul. 2005 & & 287 & -2 & Yes? & & & & \\
\hline
\end{tabular}

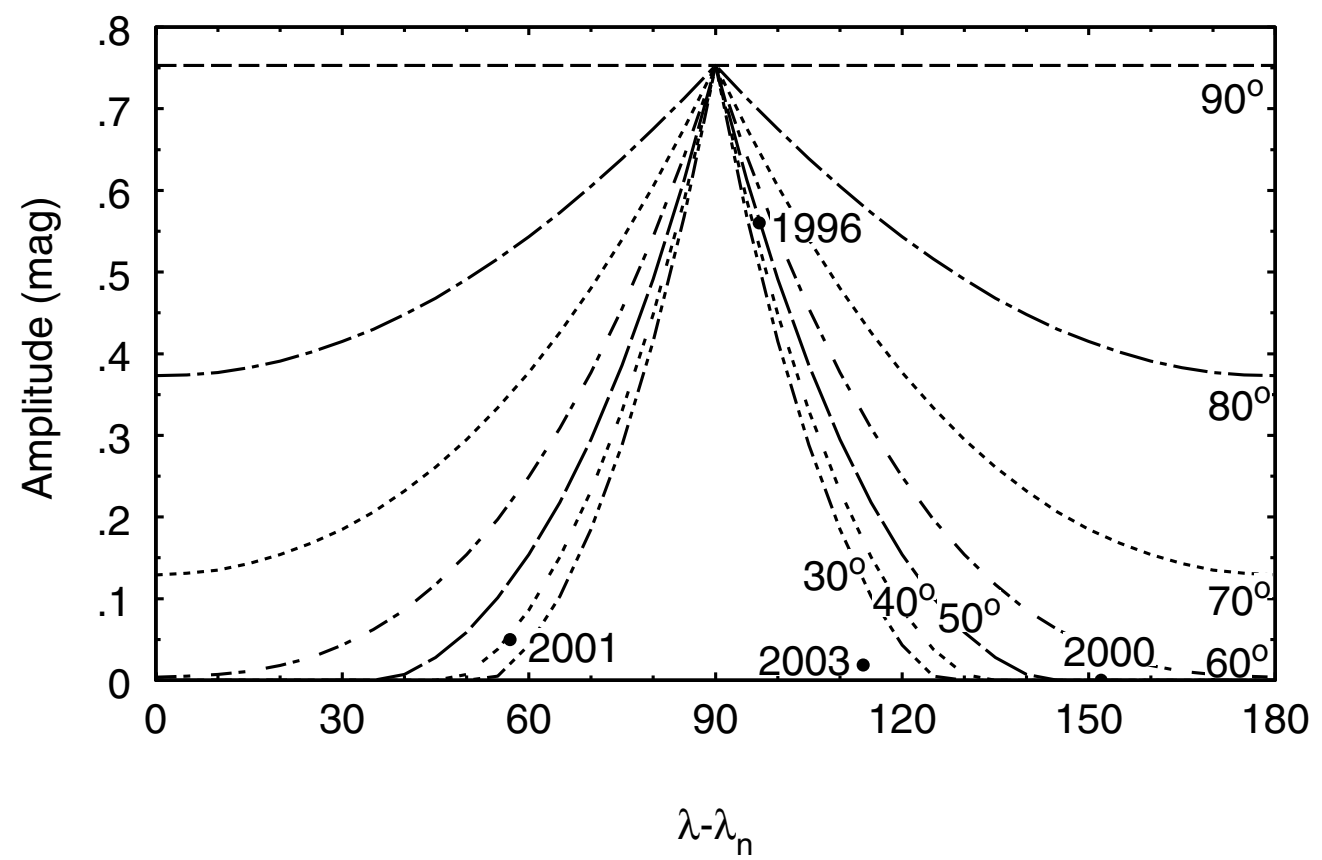

Fig. 6. Modelled amplitudes for $\beta_{n}$ (ecliptic latitude of the pole of the orbit of Antiope system) shown on the graph as functions of difference between ecliptic longitudes of the asteroid $(\lambda)$ and the orbit's pole $\left(\lambda_{n}\right)$. The observed amplitudes in 1996, 2000, 2001, and 2003 are also shown. 
nontrivial is relatively well known from the study of Kinoshita (1972). Although we have used either a simple kinematic model of motion or the numerical integration, the knowledge of exact and approximate integrals of motion proved very useful. For the shape, moments of inertia and potential of the ellipsoid, we have adopted the semi-major axis $a_{\mathrm{p}}=42.5 \mathrm{~km}$, the axes ratios $b_{\mathrm{p}} / a_{\mathrm{p}}=c_{\mathrm{p}} / a_{\mathrm{p}}=0.9$, and the density $\rho=1.3 \mathrm{~g} \mathrm{~cm}^{-3}$.

Given the attitude matrix of the ellipsoid with respect to the observer, the position of the sphere and the direction to the Sun (the latter two in the ellipsoid's reference frame) we have computed the observed magnitude using the algorithm loosely based on the principles of the so called "ray tracing method". Briefly we assume two imaginary CCD frames perpendicular to the observer-Antiope line, one for the ellipsoid and one for the sphere. For each virtual pixel we check if the ray traced from it to the asteroid hits the Sun after being reflected from the ellipsoid or a sphere. The sum of all illuminated pixels provides a simulated magnitude of the system. All mutual phenomena (occultations and eclipses) as well as phase angle effects can be reproduced in our VFM (Virtual Frames Method) code.

As suggested by the shapes of the lightcurves, at least one component of Antiope is irregular and one of the minima usually reveals a significant asymmetry. In all comparisons of the simulated lightcurve with observations, we have rejected the asymmetric minimum.

\subsection{Single period assumption}

We have started with the simplest (physically possible) model of motion. The sphere (satellite) moved around the ellipsoid (primary) on a circular orbit with the radius suitably chosen to obtain the orbital motion synchronous with the primary's rotation. The orbital plane was assumed to be perpendicular to the spin vector of the primary - the latter rotating at a constant angular rate in the short axis mode. Additionally, we fixed the satellite at the intersection of the primary's longest axis line with the orbit; the choice was justified by the fact that for the 1:1 spin-orbit resonance in the Antiope-like system such a stationary solution is stable.

Using this model we tried to determine the orientation of the rotation axis coinciding with the normal to the orbital plane. According to the assumptions, the 3-1-3 Euler angles $\varphi, \theta$, $\psi$ provide information about both the primary's attitude and the satellite's position. The symbols follow the convention of Goldstein (1980), i.e. $\varphi$ is the angle between the Equinox and the line of nodes on the Ecliptic, $\theta$ is the ecliptic colatitude of the spin vector, and $\psi$ is the rotation angle (equal the orbital argument of latitude of the satellite).

The study began with a selection of the initial values of Euler angles that lead to the occurrence of eclipses during the 1996 opposition if the period $T_{1996}=16 \mathrm{~h} .5$. Then, for each selected triplet $(\varphi, \theta, \psi)$ we varied the value of the rotation pe$\operatorname{riod} T=2 \pi / \dot{\psi}$, and simulated the lightcurves of the next three oppositions. For each opposition we compared the simulated magnitude with the observational data and evaluated the $\chi^{2}$ merit function obtained with different rotation periods.

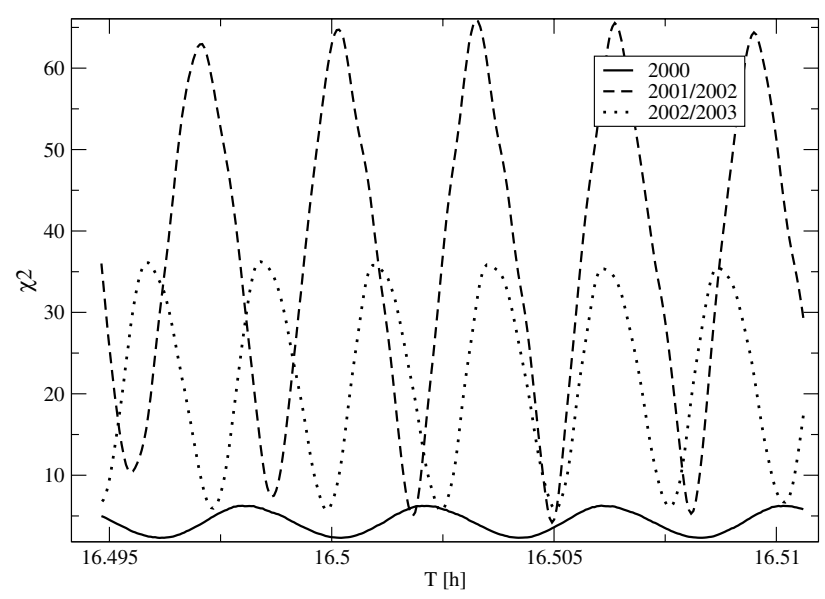

Fig. 7. $\chi^{2}$ of the simulated observations as a function of the constant rotation/orbital period for three oppositions.

Supposing the model was appropriate, the $\chi^{2}$ minima for all oppositions should occur for the same values of $T$. This was not the case, however, as shown in Fig. 7, for the oppositions 2001/02 and 2002/03, the minima of $\chi^{2}$ coincide quite well at the value of $T_{\text {orb }}=16.505$. This period was obtained with

$\varphi=90^{\circ}+\lambda_{\mathrm{n}}=107^{\circ} \pm 5$,

$\theta=90^{\circ}-\beta_{\mathrm{n}}=65^{\circ} \pm 5$,

i.e. with the ecliptic longitude of the spin vector $\lambda_{\mathrm{n}}=$ $17^{\circ}$ and its latitude $\beta_{\mathrm{n}}=25^{\circ}$. At the initial epoch $t_{0}=$ 2450438.6766 JD (1996 Dec 21.1766), the rotation angle was $\psi_{0}=95^{\circ} \pm 5$. But for the opposition 2000 the results are different, suggesting the period $T_{\text {rot }}=16.5042$. This contradicts the expectation that we can find a single period that is the best fit for all oppositions.

What is the reason for this discrepancy? Let us recall that the nature of the 2000 lightcurve is quite different from the remaining three. There is no doubt that the weak brightness variations in 2000 are due to the rotation only, whereas the remaining observations exhibit the eclipses of components. Accordingly, the results for 1996, 2001/02 and 2002/03 provide information about the orbital motion of the system, whereas the period obtained from the 2000 opposition is rather related to the rotation. An alternative explanation would require some long period precession-nutation effects.

To check the second possibility we have tilted the rotation axis with respect to the orbital momentum vector. This allows precession-nutation effects to appear (Kinoshita 1972). In spite of massive computations, we have not found any solution coherent with the observations that is physically meaningful as well; all precession periods that agreed with observations were at least 10 times too long compared to the bounds implied by the theory of Kinoshita or numerical integration.

\subsection{Almost synchronous model}

The simplest extension of the synchronous model consists of allowing the rotation rate and the orbital mean motion to be slightly different. In principle, the difference can be either systematic or periodic, depending on how far from the exact 1:1 


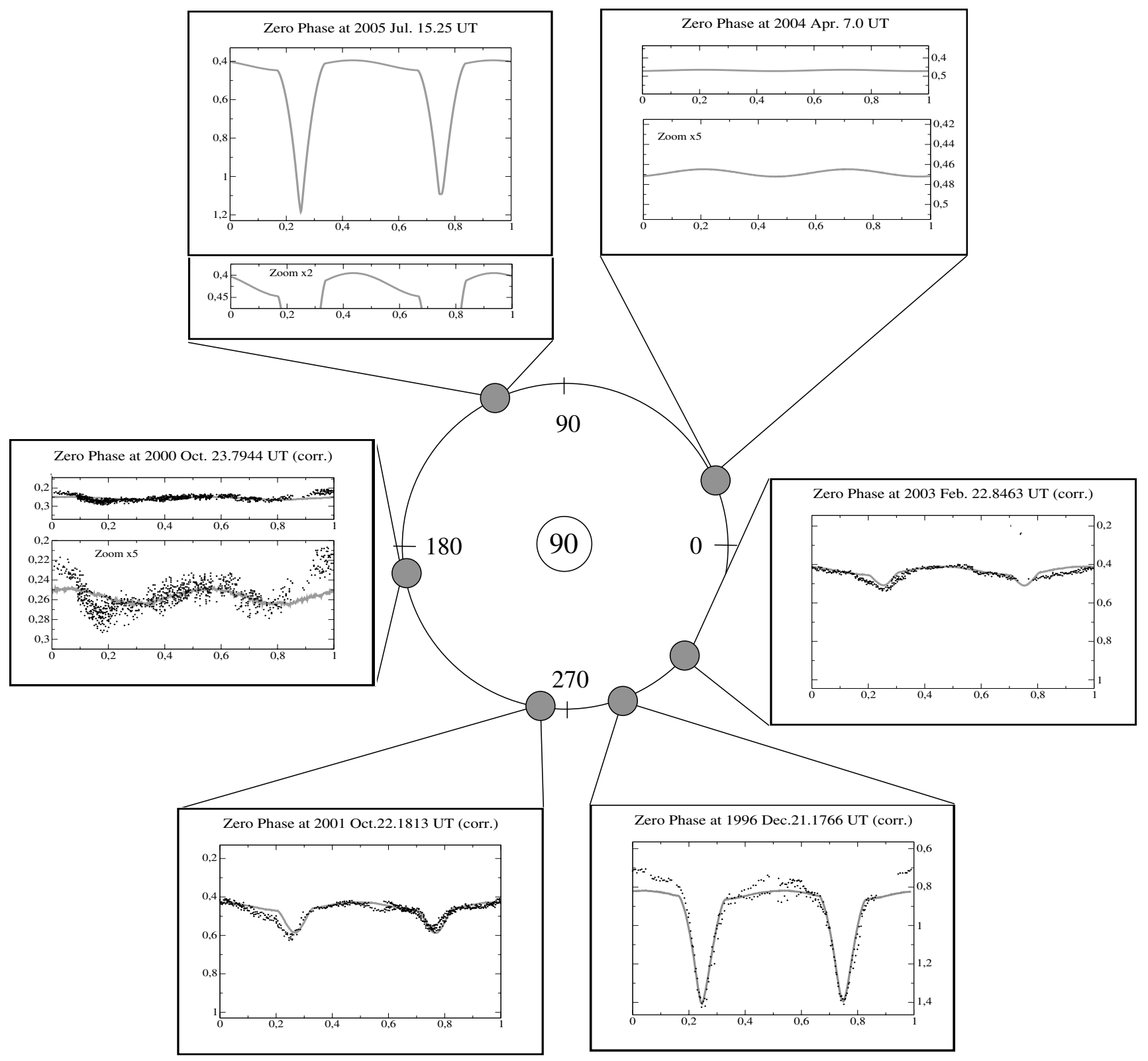

Fig. 8. The lightcurve of 90 Antiope at different oppositions. Observed values (1996 - Hansen et al. 1997; 2000, 2001, 2003 - Papers I, II, present work, respectively) are marked by dots; the lines present the results of the kinematic model with two periods.

resonance the system is. We have explored this possibility, leaving all previous assumptions (namely the alignment of the orbital and rotational momenta) unchanged. We have also assumed the same values of $\varphi$ and $\theta$. The two periods $T_{\text {orb }}$ and $T_{\text {rot }}$ obtained in the previous section served as the first approximation for the orbital and rotation periods respectively. Although Fig. 7 does not clearly favor any of the $2000 \mathrm{~min}$ ima of $\chi^{2}$, other choice of $T_{\text {rot }}$ is reflected in an incorrect offset between the rotation and eclipse extrema during the remaining oppositions.

With $\varphi$ and $\theta$ fixed, we have adjusted three parameters: the orbital period $T_{\text {orb }}$, rotation period $T_{\text {rot }}$ and the initial difference between the satellite argument of latitude and rotation angle $\delta_{0}=\theta_{0}-\psi_{0}$. In the previous section we had $\theta_{0}=\psi_{0}$; this time both angles may differ and we assumed $\theta_{0}=95^{\circ}$. Proceeding similarly to the previous case, we have determined

$T_{\text {orb }}=16.5051 \pm 0.0002$,

$T_{\text {rot }}=16.5047 \pm 0.0002$,

$\delta_{0}=10^{\circ} \pm 5$.

Figure 8 summarizes our results. The 90 Antiope is placed in the centre of the figure; the small gray circles indicate the ecliptic longitude of the Earth in the reference frame of the asteroid during the four observed and two future oppositions. To each gray circle we have attached a plot of magnitude versus the phase, where dots represent the observations (where available) and the gray line shows the synthetic lightcurve simulated by our model using the two periods $T_{\text {orb }} \neq T_{\text {rot }}$ determined in this section. The model well represents the occultations/eclipses, 
whereas the influence of the rotation of the irregularly shaped body is not accounted for due to our ellipsoid-sphere assumptions. In some instances we provide two plots for a single opposition: the upper ones maintain the common vertical scale of the magnitude axes, the lower ones are vertically stretched (zoomed) to indicate the phenomena hardly visible in the upper plots.

We have tried to predict the lightcurves for the next two oppositions. According to our model, there should be no mutual phenomena during the April 2004 opposition. In July 2005, the occultations/eclipses should be clearly visible. If our model is correct, the shift of the rotation maxima with respect to the occultation minimum should be observed. The new lightcurves from these two apparitions are required to revise the model presented here of the binary system of the asteroid 90 Antiope.

Acknowledgements. This work was partially supported by the Polish KBN Grants 2 P03D 00718 and 2 P03D 007 22. SF thanks G. Santacana, J.-M. Vugnon and M. Fauvaud for their assistance during the observations at Chateau Renard. The Borowiec observations were reduced with the CCLRS STARLINK package.

\section{References}

Behrend, R., Roy, R., Rinner, C., et al. 2004a, IAUC, 8265

Behrend, R., Roy, R., Sposetti, S., et al. 2004b, IAUC, 8292

Goldstein, H. 1980, Classical Mechanics (Reading: Addison-Wesley)

Hansen, A. T., Arentoft, T., \& Lang, K. 1997, Minor Planet Bull., 24, 17

Kinoshita, H. 1972, PASJ, 24, 423

Margot, J. L. 2003, IAUC, 8182
Margot, J. L., Nolan, M. C., Negran, V., et al. 2003, IAUC, 8227

Merline, W. J., Close, L. M., Dumas, C., et al. 2000, BAAS, 32, 1306

Merline, W.J, Weidenschilling, S. J., Durda, D. D., et al. 2002a, in Asteroids III, ed. W. F. Bottke, A. Cellino, P. Paolicchi, \& R. P. Binzel (Univ. of Arizona), 289

Merline, W. J., Tamblyn, P. M., Dumas, C., et al. 2002b, IAUC, 7980

Merline, W. J., Close, L. M., Tamblyn, P. M., et al. 2003a, IAUC, 8075

Merline, W. J., Dumas, C., Siegler, N., et al. 2003b, IAUC, 8165

Merline, W. J., Tamblyn, P. M., Dumas, C., et al. 2003c, IAUC, 8183

Merline, W. J., Tamblyn, P. M., Chapman, C. R., et al. 2003d, IAUC, 8232

Michałowski, T., Colas, F., Kwiatkowski, T., et al. 2001, A\&A, 378, L14 (Paper I)

Michałowski, T., Colas, F., Kwiatkowski, T., et al. 2002, A\&A, 396, 293 (Paper II)

Millis, R. L. 2003, IAUC, 8251

Nolan, M. C., Howell, E. S., Rivkin, A. S., \& Neish, C. D. 2003a, IAUC, 8163

Nolan, M. C., Hine, A. A., Howell, E. S., et al. 2003b, IAUC, 8220

Noll, K., Stephens, D., Grundy, W., et al. 2002, IAUC, 8034

Ostro, S. J., Nolan, M. C., Benner, L. A. M., et al. 2003, IAUC, 8237

Pravec, P., Kusnirak, P., Sarounova, L., et al. 2003a, IAUC, 8216

Pravec, P., Benner, L. A. M., Nolan, M. C., et al. 2003b, IAUC, 8244

Ryan, W. H. 2003, IAUC, 8128

Stephens, D. C., Noll, K. S., \& Grundy, W. 2004, IAUC, 8289

Tamblyn, P. M., Merline, W. J., Chapman, C. R., et al. 2004, IAUC, 8293

Veillet, C., Griffin, I. P., \& Parker, J. W. 2002, BAAS, 34, 0909

Weidenschilling, S. J., Paolicchi, P., \& Zappala, V. 1989, in Asteroids II, ed. R. P. Binzel, T. Gehrels, \& M. S. Matthews (Univ. of Arizona), 643

Weidenschilling, S. J., Marzari, F., Davis, D. R., \& Neese, C. 2001, Lunar and Planetary Science XXXII 